\title{
Interview with Vanamala Vishwanatha
}

\section{Renowned Translation Studies scholar}

Dr. Vanamala Vishwanatha speaks to NTM

on the experience of translating, studying

translations and dealing with languages.

1. You have diverse experience of teaching English Language, Translation Studies, Dalit Writing, Modern Indian Literatures, Women's Writing, Indian Writing in English, Oral \& Written Communication and Gender Studies. But, you have also specialized in Translation Studies. What attracted you towards Translation \& Translation Studies?

I was trained as an English teacher and therefore my career has been with English Studies. But I've always been fascinated by Kannada language, literature and culture. Translation, which deals with both languages, afforded me a space to bring matters of head and heart together. That is what holds me to translation. When I moved to Bangalore University in the early 90 s, I could give legitimate attention to translation as it was being taught in the Department of English as an elective course at the MA level. Further, it was also around this time that Translation Studies was beginning to emerge as a fledgling discipline. And it was so heady to be growing along with a new and exciting area. But what gave an edge to my engagement with TS is that students, especially those who hailed from rural areas, found it very engaging and empowering as the theory and practice of translation accorded value to their word and their world. And as a people who live and breathe with/in many languages, we have a lot to contribute to this field; though, its potential is yet to be realized.

2. You translate between Kannada and English. You have won KATHA award as the Best Translator of Kannada fiction into English. Do you think translating from Kannada to English is different from translating from English to Kannada? 
Yes, the two activities are vastly different as the linguistic and cultural differences between them are also enormous. While the syntactic structure of Kannada is Subject-Object-Verb (SOV) that of English is SVO. While Kannada has had a much longer history, English is relatively young. But while Kannada remains a language of the State, English has become the language of the Globe. Thus, the power equations between Kannada and English are anything but symmetrical. Therefore, one is better off looking for a theory of Non-equivalence rather than a theory of Equivalence and commensurability when translating between the two languages.

One has to safeguard the interest of Kannada, the more vulnerable language in the face of hegemonic English by breaking and blending, fracturing and fissuring standard English to reconstruct the 'strange' world of Kannada and retain its local colors and tones. But while translating from English into Kannada, one has to choose a mode to match the purpose and audience. For instance, while most of the earlier translations from English tended to trans-create the text taking substantial liberties with the text, we find that the later translations often go in for a more literalist strategy. Further, because the readership for English is so varied - local, national, international - that, often, one has no sense of an audience at all when one is translating into English. It's as though your translation goes into a black hole. Whereas, when you're translating into Kannada, there is a very definitive sense of readership. As this readership is more homogenous and familiar, it is easier to decide on the how of communicating.

\section{You have attended quite a few Summer Institutes in Linguistics and ELT, both in India and abroad. To what extent Linguistics and ELT helped you in practical Translation?}

Except for providing one with a sense of the complexity and beauty of Language, I'm afraid, an explicit knowledge of Linguistics is not much help for a translator. Same is the case with ELT. If you're a translator translating from/into English, ELT may help you to hone 
your skills better. On the contrary, translation has a lot to contribute to both disciplines, an issue that has tremendous research potential.

4. How was the experience of translating a feminist writer in Kannada like Sara Aboobacker and introducing her to the English speaking world?

It has been a very fulfilling experience as the text has had a rich 'afterlife' in English translation. The text has received a lot of attention by way of being prescribed in courses on Translation, Gender and General English. Several dissertations have also used this resource. I've had many students and teachers saying how moving the story was. Many an upcoming writer has commented on the nuanced use of language in the translation. The notion of 'translation activism' (using translation as a means of questioning the status quo and creating a different canon) that underlies the whole enterprise has been appreciated widely within Translation Studies, leading to a couple of close studies of the text. It has contributed to current feminist scholarship by introducing the writing of a first generation, literate woman from a marginalized community.

5. You have worked with International Translation Studies scholar Prof. Sherry Simon, Concordia University, Canada for a collaborative project on 'Translation Studies \& Kannada'. How was the experience? What kind of academic or cultural exchange did you have with her?

Prof. Simon, a true Indianist, came down to Bangalore in 1996 on her sabbatical. We worked together on BM Srikanthaiah and A K Ramanujan, which we wrote up as an essay for "Post-colonial Translation', edited by Bassnett \& Trivedi. We also translated a short story called 'Devi' by $\mathrm{P}$ Lankesh and interviewed two eminent translators-Ramachandra Sharma and H S Shiva Prakash, along with publishing an article on the politics of translation reviews for 'The Hindu'. Given that Translation was a common area of interest, we together forged a more historically-grounded approach to the study of translations in the Kannada context. 
6. You have been associated with Krishnamurthi Foundation, India. You have co-edited many books of the foundation with Prof.O.L.Nagabhushana Swamy and others. It is said that it is quite difficult to translate $J$ Krishnamurti into any language. What are the challenges involved in translating $\mathrm{J}$ Krishnamurthy into Indian languages?

JK is no more difficult than translating any other philosopher. One of the challenges in translating him is that he often provides an extra charge to ordinary words like 'intelligence' or 'awareness'. Therefore we were faced with the question - should we or should we not provide a glossary for these words. But we decided against it as we felt that readers will get the meaning in the immediate context of the text as well as from the overall movement of JK's thought. Secondly, JK wrote in rebellion against organized religion; but the language of spirituality in Kannada has emerged out of that kind of context. So the challenge for us was to see how we could express JK's thought without recourse to a well-established religious register in Kannada. There have been others which Prof.O L Nagabhusana Swamy and Prof.H S Raghavendra Rao who have handled the bulk of these translations will be able to comment on.

7. You have served as Hon. Director of Centre for Translation, Central Sahitya Akademi, Bangalore. What kind of initiatives have you taken to promote regional language literature through translation?

When I took over as Director, the Centre had the larger view of translating ancient classics from the four South Indian languages into other Indian languages and English. Apart from completing and publishing 17 of these texts within two years, the highlight for me was the publication of Shivarama Karantha's modern Kannada classic 'Marali Mannige' as 'Return to Earth', ably and painstakingly translated by Smt. Padma Ramachandra Sharma, with a perceptive introduction by Prof.Rajendra Chenni, We were able to organize the launch of a forum for translation called 'Translation Matters' in 
Hyderabad with enthusiastic support from Prof.Alladi Uma, Prof. Sridhar and Dr.Suneetha Rani of Central University, Hyderabad.

8. You are also a member of the Project Advisory Committee of National Translation Mission (NTM). As you know, probably NTM is the first gigantic initiative of its kind in the world to undertake such a stupendous task of translating knowledge texts in 69 disciplines into 22 languages. Do you think knowledge text translation has been overlooked by our translators?

While there were specific institutions such as the Sahitya Akademi at the centre and its counterparts in each language which promoted the translation of literary texts, there have not been similar bodies exclusively meant to plan and carry out the translation of knowledge texts with a vision. Though there have been some attempts from institutions devoted to the Sciences and the Social Sciences, the translation of knowledge texts has been, by and large, left to individual initiatives and private commercial interests like the 'Student Guides'. Therefore, there has been a crying need for such translations. If we want to be true to our democratic ideal of providing equitable access to education for all our people, we need to pursue this goal assiduously.

\section{Though some of the state and central universities have opened departments of TS, we still do not have enough centres in our country. What do you think needs to be done to promote TS?}

Translation demands a lot - the knowledge of two languages, readership, cultures along with the knowledge of specific domains. But our tertiary and higher education Institutions have done a positive disservice to the teaching and learning of Indian languages by not giving them the attention they deserve. Further, the global reach and power of English has not helped the cause of regional languages either. Therefore, we need to strengthen the foundations of Language Education and equally, devise creative courses for training professional translators. 
10. As the Central Government has set up a body like NTM, the need and scope of translation is quite evident now. What kind of support from academic institutions would promote the cause of the mission?

Since domain knowledge is imperative for NTM's goal of producing the translations of knowledge texts, academic institutions, have a seminal role to play in helping NTM produce quality translations. So this partnership is crucial for translating NTM's vision.

Interview by. Shashikumar J

March 2012 